\title{
Experimental Study of the Magnetocaloric Effect in the Two-Dimensional Quantum System $\mathrm{Cu}(\boldsymbol{t n}) \mathrm{Cl}_{2}$
}

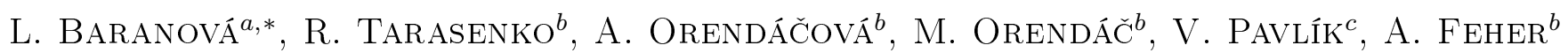

${ }^{a}$ Institute of Construction, Technology and Management, Department of Applied Mathematics, Civil Engineering

Faculty, Technical University of Košice, Vysokoškolská 4, 04200 Košice, Slovakia

${ }^{b}$ Institute of Physics, Faculty of Science, P.J. Šafárik University, Park Angelinum 9, 04154 Košice, Slovakia

${ }^{c}$ Institute of Experimental Physics of SAS, Watsonova 47, 04001 Košice, Slovakia

\begin{abstract}
Magnetocaloric studies of a two-dimensional antiferromagnet $\mathrm{Cu}(t n) \mathrm{Cl}_{2}(t n=1,3$ - diaminopropane $=$ $\mathrm{C}_{3} \mathrm{H}_{10} \mathrm{~N}_{2}$ ) have been performed by adiabatic magnetization and demagnetization measurements, in the temperature range from 0.2 to $4 \mathrm{~K}$ and magnetic fields up to $2 \mathrm{~T}$. The compound represents an $S=1 / 2$ spatially anisotropic triangular-lattice antiferromagnet. The magnetocaloric measurements were focused at the identification of the phase transition to the magnetically ordered state which was not indicated in the previous specific heat studies. Furthermore, the interplay of the magnetic-field induced easy-plane anisotropy and the intrinsic spin anisotropy present in the studied system should manifest in low magnetic fields. The obtained results of the magnetocaloric experiments of $\mathrm{Cu}(t n) \mathrm{Cl}_{2}$ indicate a double crossover from the normal to inverse magnetocaloric effect (MCE). The first crossover from the normal to inverse MCE occurring at about $0.3 \mathrm{~K}$ can be attributed to the competition of the aforementioned anisotropies. The second crossover from the inverse to normal MCE observed at about $2.2 \mathrm{~K}$ might be ascribed to the formation of spin vortices stabilized by the easy-plane anisotropy introduced by magnetic field.
\end{abstract}

DOI: 10.12693 /APhysPolA.126.256

PACS: 75.50.Ee, 75.30.Sg

\section{Introduction}

Two-dimensional (2D) quantum antiferromagnets have attracted a significant amount of theoretical and experimental attention due to the unconventional magnetic properties resulting from the interplay between quantum fluctuations and geometrical frustration [1].

The title compound has been previously identified as an $S=1 / 2$ spatially anisotropic triangular antiferromagnet from the collinear Néel phase realized with dominant interactions forming a square-lattice pattern [2]. For $\mathrm{Cu}(t n) \mathrm{Cl}_{2}$ studied in $B=0$, no evidence of longrange magnetic order was observed. The data analysis suggested intralayer antiferromagnetic nearest-neighbor $\left(J / k_{\mathrm{B}}=3 \mathrm{~K}\right)$, frustrating next-nearest-neighbor $(0<$ $\left.J^{\prime} / J<0.6\right)$, and interlayer $\left(\left|J^{\prime \prime} / J\right| \approx 10^{-3}\right)$ interactions. Thermodynamic studies identified the response of $\mathrm{Cu}(t n) \mathrm{Cl}_{2}$ in nonzero magnetic field as a field-induced Berezinskii-Kosterlitz-Thouless (BKT) phase transition below $1 \mathrm{~K}[3]$.

In this paper, the magnetocaloric study of polycrystalline $\mathrm{Cu}(t n) \mathrm{Cl}_{2}$, investigated at low temperatures is presented. The magnetocaloric effect is a useful and sensitive technique for mapping magnetic phase transitions as well as for understanding the nature of magnetic transitions [4]. The MCE study was used to trace the phase transition in $\mathrm{Cu}(t n) \mathrm{Cl}_{2}$ expected at $0.8 \mathrm{~K}$ [3].

*corresponding author; e-mail: lucia.baranova@tuke.sk

\section{Experimental details}

Magnetocaloric study was performed on powdered polycrystals pressed into the pellet of $\mathrm{Cu}(t n) \mathrm{Cl}_{2}$ with a mass of $10 \mathrm{mg}$ in a commercial dilution ${ }^{3} \mathrm{He}^{-}{ }^{4} \mathrm{He}$ refrigerator TLE 200 in the temperature range from 0.2 to $4 \mathrm{~K}$ in magnetic fields, $B$, up to $2 \mathrm{~T}$. Magnetocaloric effect performed by adiabatic magnetization and adiabatic demagnetization measurements was measured using a direct method.

The sample was placed in a holder using nylon fibers with a diameter of $0.1 \mathrm{~mm}$. A $\mathrm{RuO}_{2}$ resistance thermometer of type RVP 575 with a nominal value of 4.7 $\mathrm{k} \Omega$ was glued to the sample using varnish GE 7031 . The nylon fibers provided a very poor thermal contact between the sample and a thermal reservoir. This thermal contact was used for initial cooling of the sample.

The crystal structure of $\mathrm{Cu}(t n) \mathrm{Cl}_{2}$, established at $150 \mathrm{~K}$, is orthorhombic (space group Pna21) with the lattice parameters $a=17.956 \AA, b=6.859 \AA$, and $c=5.710 \AA[2]$. The structure consists of covalently bonded ladders running along the $c$-axis, while the adjacent ladders in the $b c$-plane are linked through intermolecular $\mathrm{N}-\mathrm{H}$... Cl hydrogen bonds formed by all four $\mathrm{H}$ atoms of the amino groups. In the $a$ direction, the layers are connected by weak $\mathrm{C}-\mathrm{H} \cdots \mathrm{Cl}$ type interactions.

\section{Results and discussion}

In the first step of magnetocaloric measurements, the sample temperature was stabilized in zero magnetic field at the initial value, $T_{0}$. Subsequently, the magnetic field was swept to the required value in the quasi-adiabatic conditions with a sweeping rate $100 \mathrm{mT} / \mathrm{min}$. The appli- 
cation of magnetic field in adiabatic conditions $(\Delta S=0)$ leads to the change of the sample temperature from $T_{0}$ to $T_{B}$. The variation of sample temperature $T_{B}$ measured from various initial temperatures $T_{0}$ is presented in Fig. 1.

When the initial temperature $T_{0} \leq 0.27 \mathrm{~K}$, the change $\Delta T=T_{B}-T_{0}$ is positive in all magnetic fields up to $2 \mathrm{~T}$. This behaviour is characteristic for a normal MCE typical for standard paramagnets. However, further increase of initial temperature above $0.27 \mathrm{~K}$ leads to the appearance of inverse $\mathrm{MCE}$ ( $\Delta T$ is negative) persisting up to $T_{0} \approx$ $2.5 \mathrm{~K}$. At higher temperatures, the normal MCE was observed again as expected for a classical paramagnet.

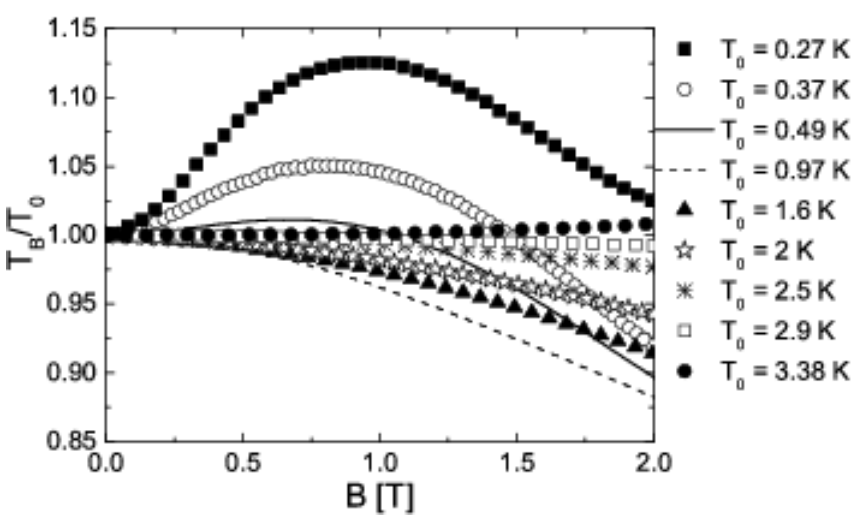

Fig. 1. Magnetic-field dependence of sample temperature during quasi-adiabatic magnetizing of $\mathrm{Cu}(t n) \mathrm{Cl}_{2}$. The temperature $T_{B}$ is normalized by initial temperature $T_{0}$.

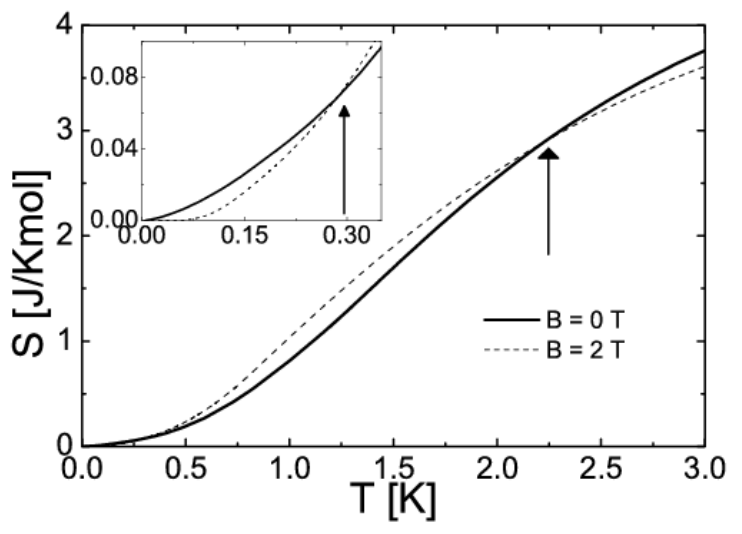

Fig. 2. Temperature dependence of the total entropy of $\mathrm{Cu}(t n) \mathrm{Cl}_{2}$ in zero magnetic field and magnetic field of $2 \mathrm{~T}$. Inset: The detail view of the low-temperature dependence of the entropy. Arrows denote the crossovers.

Observation of normal and inverse magnetocaloric effect in $\mathrm{Cu}(t n) \mathrm{Cl}_{2}$ is consistent with the behaviour of the entropy of the studied system in zero magnetic field and magnetic field of $2 \mathrm{~T}$. Both curves of the entropy (Fig. 2) were obtained by integrating the total specific heat of $\mathrm{Cu}(t n) \mathrm{Cl}_{2}[5]$.
Below the temperature $T \approx 0.3 \mathrm{~K}$, the entropy in magnetic field of $2 \mathrm{~T}$ is lower than the zero field entropy, which results in the normal MCE. In the temperature range $0.3 \mathrm{~K}<T<2.25 \mathrm{~K}$, the entropy curve in $B=2 \mathrm{~T}$ is above the curve of the entropy in $B=0$, which leads to the inverse MCE. Finally, above the temperature $T \approx 2.25 \mathrm{~K}$, the entropy in $B=0$ is bigger than the entropy in $B=2 \mathrm{~T}$, which manifests the normal MCE.

The comparison of the experimental data of adiabatic temperature changes $T_{B}$ (Fig. 1) and temperature dependence of the entropy (Fig. 2) yielded relatively good agreement in observed crossovers between the normal and inverse $\mathrm{MCE}$ in $\mathrm{Cu}(t n) \mathrm{Cl}_{2}$. As can be seen from Fig. 1 and Fig. 2, no crossover in MCE has been observed around the temperature $0.8 \mathrm{~K}$. This behaviour indicates the absence of the long-range order in $\mathrm{Cu}(t n) \mathrm{Cl}_{2}$ in the expected temperature region.

As was shown in Ref. [3], applied magnetic field induced easy-plane anisotropy in $\mathrm{Cu}(t n) \mathrm{Cl}_{2}$, resulting in the BKT transition, which causes reduction of the entropy in non zero magnetic field. Correspondingly, the first crossover from the normal to the inverse MCE, occurring at about $0.3 \mathrm{~K}$ (Fig. 2), can be attributed to the competition of the magnetic-field induced easy-plane anisotropy and the intrinsic spin anisotropy present in the studied system. The second crossover from the inverse to normal MCE, observed at about $2.2 \mathrm{~K}$, might be ascribed to the formation of spin vortices stabilized by the easy-plane anisotropy introduced by the magnetic field.

\section{Conclusions}

Magnetocaloric study has been performed on a $2 \mathrm{D}$ quantum system $\mathrm{Cu}(t n) \mathrm{Cl}_{2}$ at temperatures below $4 \mathrm{~K}$ in magnetic fields up to $2 \mathrm{~T}$. A double crossover from the normal to the inverse MCE has been attributed to the formation of vortices stabilized by the easy-plane anisotropy, induced by the applied magnetic field. In future, further studies are desirable, to estimate the kind and the strength of the intrinsic spin anisotropy.

\section{Acknowledgments}

This work was supported by the projects VEGA 1/0143/13, ITMS 26220120005 and APVV LPP-0202-09.

\section{References}

[1] S. Sachdev, Nat. Phys 4, 173 (2008).

[2] V. Zeleňák, A. Orendáčová, I. Č́sařová, J. Černák, O.V. Kravchyna, J.-H. Park, M. Orendáč, A.G. Anders, A. Feher, M.W. Meisel, Inorg. Chem 45, 1774 (2006).

[3] A. Orendáčová, E. Čižmár, L. Sedláková, J. Hanko, M. Kajňaková, M. Orendáč, A. Feher, J.S. Xia, L. Yin, D.M. Pajerowski, M.W. Meisel, V. Zeleňák, S. Zvyagin, J. Wosnitza, Phys. Rev. B 80, 144418 (2009).

[4] R. Rawat, I. Das, Phys. Rev. B 64, 052407 (2001).

[5] L. Sedláková, Ph.D. Thesis, P.J. Šafárik University, Košice 2010. 